\title{
Experiência de Clínica Ampliada em Odontologia na Universidade Estadual de Maringá
}

\author{
An Experience with Expanded Clinical Care in \\ Dentistry at the State University in Maringá
}

\author{
Mitsue Fujimaki Hayacibaral \\ Luiz Fernando Lolli ${ }^{I}$ \\ Raquel Sano Suga Terada \\ Mirian Marubayashi Hidalgo \\ Carina Gisele Costa Bispo \\ Hélio Hisashi Terada \\ Luciene Silvério Padilha \\ Julyana Karolina Rodrigues ${ }^{I}$ \\ Arivaldo de Jesus Vicente \\ Aline Cláudia Ribeiro Medeiros ${ }^{I}$ \\ Cristiane Muller Calazans ${ }^{I}$
}

\section{PALAVRAS-CHAVE:}

- Humanização da Assistência;

- Sistema Único de Saúde;

- Odontologia.

\section{KEYWORDS: \\ - Assistance Humanization; \\ - Brazilian Health System; \\ - Dentistry.}

Recebido em: 20/09/2010

Aprovado em: 30/11/2010

\section{RESUMO}

A clínica ampliada (CA) representa uma estratégia da Política Nacional de Humanização (PNH) para reflexão sobre o cuidado com o usuário do Sistema Único de Saúde (SUS). Impulsionados pelo Programa Pró-Saúde e pela PNH, docentes do curso de Odontologia da UEM promoveram uma reestruturação do atendimento clínico ofertado, resultando na implantação da CA. Este trabalho relata a experiência vivenciada na implantação dessa clínica, que visa a promover a interação e o vínculo do aluno com a comunidade e a equipe multiprofissional de saúde, de modo a obter o cuidado integral e humanizado. A CA está estruturada para acolher o usuário em um ambiente multiprofissional, direcionando-o dentro de um fluxograma até a sua alta, promovendo a autonomia na manutenção de sua saúde bucal. A CA tem dado subsídios para melhorar a visão do cuidado integral do usuário da clínica odontológica da UEM, fortalecendo a formação acadêmica e o cuidado prestado à comunidade. Nota-se que a construção coletiva agrega muito valor às ações, mas é um processo lento e depende da maturidade dos envolvidos.

\section{ABSTRACT}

Expanded clinical care is a strategy under the National Policy for Humanized Care for reflection on care for users of the Unified National Health System. Under the impetus of the Pró-Saúde program and the National Policy for Humanized Care, faculty from the School of Dentistry at the State University in Maringá (UEM) conducted a restructuring of clinical care, resulting in the implementation of expanded care. This study reports on the experience with the implementation of such expanded clinical care, aimed at promoting interaction between students, community, and the multi-professional health team, so as to obtain comprehensive and humanized care. Expanded care is structured to receive users in a multi-professional setting, directing them within a flowchart all the way to their discharge and fostering autonomy in the maintenance of their oral health. Expanded care has provided backing for improving the vision of comprehensive care for users of the UEM dental clinic, strengthening academic training and community care. The collective construction adds considerable value to the activities, but it is a slow process that requires maturity on the part of participants. 


\section{INTRODUÇÃO}

A Política Nacional de Humanização (PNH) surgiu no ano de 2004 trazendo a prerrogativa de avançar o Sistema Único de Saúde (SUS) por meio da valorização das dimensões subjetiva e social em todas as práticas de atenção e gestão, pelo estímulo a processos comprometidos com a produção de saúde, fortalecimento do trabalho em equipe multiprofissional e pela utilização da informação, da comunicação, da educação permanente e dos espaços de gestão na construção da autonomia e do protagonismo de sujeitos e coletivos ${ }^{1}$. No universo das ações previstas na PNH está a clínica ampliada (CA). O termo ampliada foi empregado para dimensionar a atuação clínica para além das abordagens convencionais que protocolizam o atendimento aos usuários, restringem seus sentimentos, ignoram seus valores e saberes além de ofuscar suas expectativas. A CA é a redefinição de trabalho da verticalização da assistência individual, familiar ou coletiva. Ela considera que o "objeto de trabalho" da assistência à saúde é uma pessoa, um grupo, uma família ou um coletivo institucional com doença ou risco de adoecer. O novo modelo de trabalho, portanto, transpõe a clínica tradicional, que tem como foco somente a doença ${ }^{2}$.

A filosofia da CA se associa ao projeto pedagógico do curso de Odontologia da Universidade Estadual de Maringá (UEM), haja vista que este tem como meta a formação de um cirurgião-dentista generalista, centrado nas necessidades de saúde da população e na política de saúde vigente no País. Dentre as ações desenvolvidas no referido curso, destaca-se a inserção dos acadêmicos na Estratégia Saúde da Família (ESF) em uma comunidade de alto risco social. As atividades direcionadas à comunidade vão desde visitas domiciliares, atividades coletivas de promoção de saúde, programas preventivos em equipamentos sociais, participação em reuniões da equipe da ESF, participação efetiva no Conselho Local de Saúde e atendimento odontológico. A inserção dos acadêmicos na realidade de vida das pessoas permitiu reflexões diversas sobre os determinantes do processo saúde-doença. Percebeu-se que muitas doenças carregam fortes conotações psicológicas, afetivas e sociais na sua gênese, fatores esses que dificultam a reabilitação dos pacientes, mesmo sendo submetidos a tratamentos aparentemente eficazes. Entretanto, foi possível verificar que tal diagnóstico não é suficiente no "campo de estágio". Observou-se a necessidade de trazer essa compreensão e trabalhar a realidade de vida na prática clínica, mudando o foco da doença para a produção de saúde. Nesse contexto, a adesão do curso de Odontologia da UEM ao Programa Nacional de Reorientação da Formação Profissional em Saúde (Pró-Saúde) desenvolveu uma dinâmica diferente no curso e promoveu maior integração dos docentes no planejamento de ações, maior entendimento dos princípios do SUS e percepção da necessidade da formação para o sistema. Essa articulação entre saber, sujeitos e ideias aliada à boa condução dos trabalhos da equipe e ao respeito e valorização do ser humano nos processos de trabalho, estes com base na $\mathrm{PNH}$, transformou o clima organizacional, possibilitou ricos momentos de discussão e criou necessidades de mudança, fatores que resultaram na construção coletiva da clínica ampliada.

Assim, no presente trabalho, apresentar-se-á a experiência de implantação da clínica ampliada no curso de Odontologia da UEM.

\section{RELATO DA EXPERIÊNCIA}

Desde 1992, com a implantação do Currículo Integrado, o corpo docente do curso de Odontologia da UEM, juntamente com alunos e funcionários, vem tentando operacionalizar uma proposta inovadora, testando algumas experiências, ora avançando, ora retrocedendo, mas sempre perseverando no desafio do atendimento em clínica integrada.

Em 2006, os acadêmicos iniciaram, sob orientação docente, atividades de territorialização no grupo da ESF. Em 2007, além das visitas domiciliares como um recurso importante de acesso à dinâmica familiar, incluiu-se também o atendimento em clínica dessas famílias. Com o passar do tempo, a condução do atendimento clínico foi permitindo que cada paciente fosse se tornando mais autônomo e corresponsável pela própria saúde, a partir da compreensão de sua doença como única. Os casos clínicos passaram a ser discutidos em grupos de estudos, para pensar na rede social e familiar que conforma o sujeito, procurando alternativas e soluções para os seus problemas.

Com a inclusão do curso de Odontologia no programa Pró-Saúde, houve maior frequência nos encontros da equipe de trabalho, os quais passaram a ser semanais em função da necessidade de implementação de várias ações do programa. Com isso, o processo de construção coletiva foi fortalecido. Também contribuiu significativamente para a implantação da clínica ampliada, um projeto piloto desenvolvido pelo Programa de Educação Tutorial (PET-Odontologia) de atendimento clínico por grupo de alunos segundo grau crescente de complexidade, que possibilitou a experiência dos alunos do quinto ano na inicialmente denominada "Clínica do PSF" e as várias oficinas com a comunidade, com o serviço e convidados.

A CA do curso de Odontologia da UEM tem como objetivo promover a interação e o vínculo do aluno com os sujeitos envolvidos (comunidade, equipe multiprofissional de saúde, 
academia) visando a um cuidado integral e humanizado, bem como servir de referência para o atendimento odontológico, aprimorando a formação do cirurgião-dentista e a assistência prestada aos usuários do SUS. O atendimento odontológico na clínica é iniciado conjuntamente pelos acadêmicos do quinto e terceiro anos, apoiados por uma equipe multidisciplinar de docentes, em que diferentes planos de tratamento são realizados, oportunizando aprendizado ao aluno e opções ao usuário, que escolhe aquele que melhor representa seus anseios e condições. A partir desse planejamento, o usuário segue um fluxo de acordo com o grau de complexidade dos procedimentos e é direcionado para o acadêmico habilitado para o atendimento.

Essas mudanças são imprescindíveis para a evolução da parte prática do currículo integrado do curso, servindo também de referência para que outros cursos possam avaliar, rediscutir e, quiçá, implementar ou reformular seu projeto pedagógico.
A parte operacional da CA está assim organizada:

1) Início do acolhimento: o primeiro contado, pelo telefone, é feito pelo Setor de Acolhimento, anteriormente denominado Triagem, para agendamento e, nesse momento, o paciente é informado sobre algumas normas do atendimento. A porta de entrada acontece pelo sistema de referência e contrarreferência das Unidades Básicas de Saúde (UBSs) ou dos municípios da $15^{a}$ Regional de Saúde de Maringá, ou ainda casos de interesse acadêmico.

2) Primeiro atendimento: o primeiro documento a ser preenchido na chegada do paciente é o "Prontuário Clínico Único", no qual o docente e o aluno deverão assinar os planos de tratamento, e o usuário assinará a autorização para o diagnóstico e realização dos procedimentos. Todos assinam o relatório das atividades diárias. O acolhimento, o diagnóstico e os planos de tratamento são realizados conjuntamente entre alunos

FIGURA 6

Fluxograma da clínica ampliada do curso de Odontologia da Universidade Estadual de Maringá.

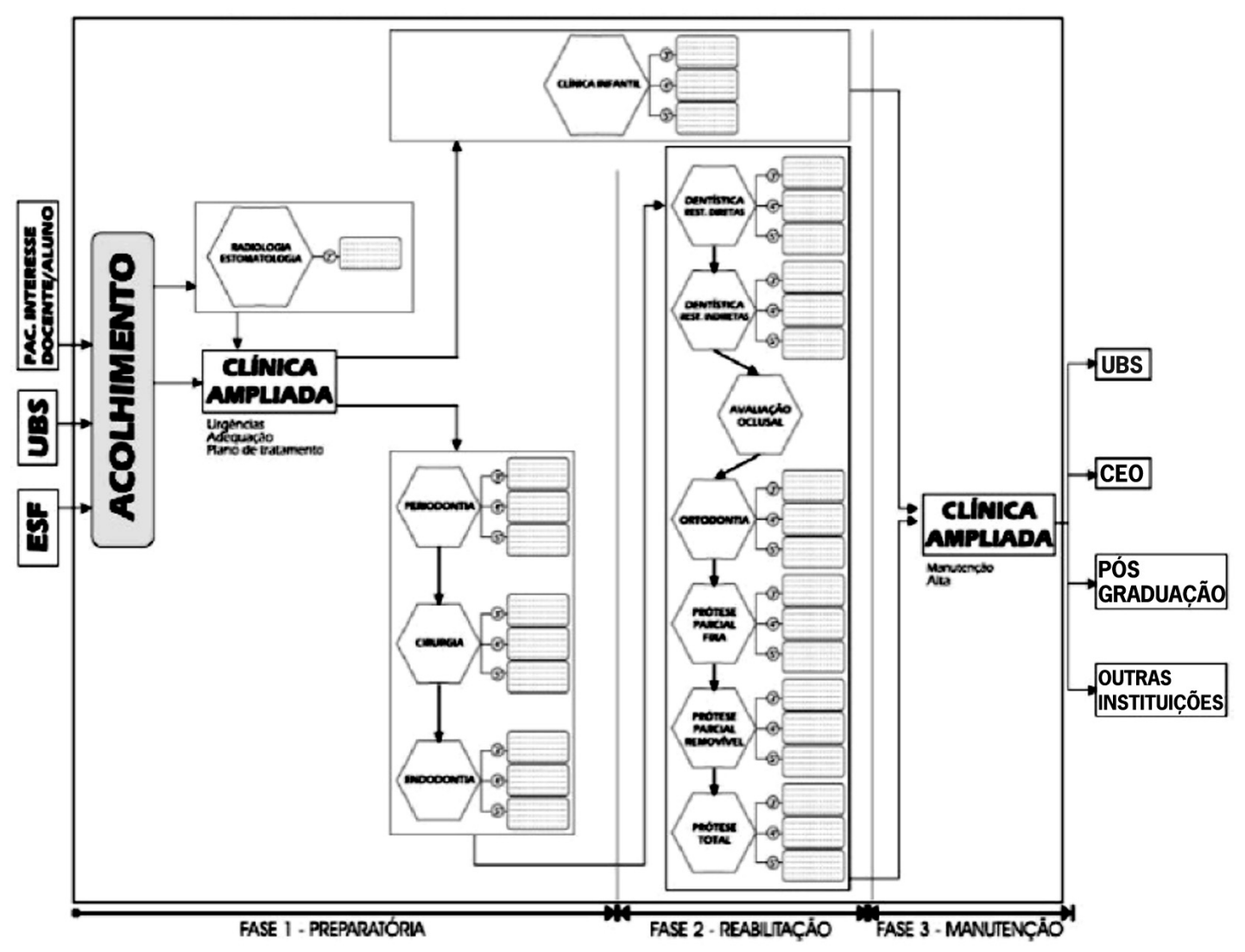


do terceiro e quinto anos, sob orientação de uma equipe de referência, da qual participam docentes de todas as áreas da Odontologia.

3) Cartão para atendimento: o estudante deverá preencher o cartão, que deverá ser apresentado pelo paciente nas próximas consultas.

4) Demais atendimentos: após a finalização de uma etapa do tratamento, o usuário será encaminhado, conforme a sua necessidade de tratamento e disponibilidade, para a distribuição das clínicas, segundo fluxograma apresentado a seguir, sendo as clínicas divididas em grau crescente de complexidade.

5) Alta: quando finalizados todos os procedimentos determinados no plano de tratamento em nível de graduação, os pacientes são novamente encaminhados para a CA para manutenção específica ou alta. No momento da alta, o usuário é encaminhado para a UBS de origem para a manutenção da condição bucal. Além disso, o usuário preenche um questionário sobre a satisfação do atendimento.

O início das atividades dessa CA aconteceu em meados de 2009 e, por isso, os resultados ainda são preliminares. Entretanto, as reuniões semanais dos docentes e funcionários que agora formalizaram uma Comissão Permanente de Avaliação da Clínica Ampliada (Copaca) têm sido mantidas para pronta avaliação, intervenção e reorientação das atividades e metas da clínica. No início de 2010, foi construído um Manual da Clínica Ampliada, para a orientação e reflexão sobre os propósitos desse trabalho ${ }^{3}$. Em termos de avaliação, foram realizadas oficinas e reuniões diversas com os participantes e segmentos envolvidos. Com base nos depoimentos dos acadêmicos do terceiro, quarto e quinto anos, que já vivenciaram o trabalho, é possível afirmar que foi muito gratificante para o estudante conseguir atender de forma ampla e completa seu paciente, proporcionando-lhe satisfação funcional e emocional, contribuindo sobremaneira para a sua qualidade de vida.

Além das vantagens já mencionadas, a CA cria um momento rico de integração e troca de conhecimentos entre os acadêmicos das três séries $\left(3^{\circ}, 4^{\circ}\right.$ e $\left.5^{\circ}\right)$, situações que lhes serão úteis na vida profissional. Um outro avanço significativo alcançado foi a elaboração do "Prontuário Clínico Único", construído coletivamente e que já se encontra em utilização, buscando traçar o projeto terapêutico singular, visando à integralidade da atenção, pensando na referência e contrarreferência com o município, e também vislumbrando o encaminhamento para outros níveis de atenção, como o Centro de Especialidades Odontológicas.

\section{DISCUSSÃO}

"Um galo sozinho não tece uma manhã: ele precisará sempre de outros galos". O trecho do texto de João Cabral de Melo Neto $^{4}$ é o que melhor explica a intenção da CA implantada no curso de Odontologia da UEM: um novo modo de trabalho que busca a integração e, consequentemente, uma melhor postura na atenção aos pacientes, uma vez que as especialidades estão integradas para um atendimento completo e diferenciado.

Após mais de duas décadas da institucionalização do SUS, existem muitos desafios a serem superados. O processo de formação em saúde evidencia que o cuidado ao paciente e ao sofrimento causado pela doença é a principal motivação na escolha dos cursos pelos alunos, devido ao conceito de que ser um profissional de saúde é principalmente tratar doenças, e não promover saúde, e à dificuldade de enxergar e entender que muitos pacientes possuem necessidades que não estão obrigatoriamente atreladas à doença e às suas consequências ${ }^{5}$. Aprender a lidar com o ser humano, e não somente com um objeto de estudo e/ou trabalho, é um desafio para a saúde. O conceito de CA vem ao encontro do novo enfoque humanizado da forma de trabalhar e consiste em um compromisso radical com o sujeito doente visto de modo singular; assume a responsabilidade sobre os usuários dos serviços de saúde; busca ajuda em outros setores, ao que se dá o nome de intersetorialidade; reconhece os limites do conhecimento dos profissionais da saúde e das tecnologias por eles empregadas; busca outros conhecimentos em diferentes setores e assume um compromisso ético profundo ${ }^{6}$.

Segundo o documento Clínica Ampliada e Compartilhada, do Ministério da Saúde ${ }^{7}$, a proposta de CA engloba como eixos fundamentais a compreensão ampliada do processo saúde-doença, entendendo que são vários os determinantes desse processo, tais como: construção compartilhada dos diagnósticos e terapêuticas, pois o reconhecimento da complexidade deve significar a compreensão da necessidade de compartilhar diagnósticos de problemas e propostas de solução; ampliação do "objeto de trabalho", com a visualização do indivíduo como um todo; transformação dos "meios" ou "instrumentos" de trabalho, melhorando arranjos e dispositivos de gestão que prestigiem a comunicação transversal; suporte para os profissionais da saúde, para que eles possam enfrentar as próprias dificuldades diante do novo. É nesse sentido que a proposta da CA do curso de Odontologia da UEM pretende seguir. Pretende ser um instrumento para que os acadêmicos possam enxergar e atuar na clínica para além de uma visão focada na boca do sujeito, sem deixar de reconhecer e utilizar o potencial de seu saber. Tal desafio de lidar com os usuários enquanto sujeitos, buscando sua participação e autonomia no 
projeto terapêutico, é tão mais importante quanto mais longo for o segmento do tratamento e maior for a necessidade de participação e adesão do sujeito no seu projeto terapêutico ${ }^{8}$.

Com a experiência da CA, alguns avanços já foram detectados, como: realização de uma anamnese ampliada; mais atenção às queixas dos pacientes; e conhecimento da história do paciente e do ambiente no qual ele está inserido. Tais condutas têm, portanto, contribuído para a detecção e o entendimento dos problemas de cada sujeito. A criação de um vínculo maior com o paciente é essencial, sendo necessário aprender a prestar atenção nesses fluxos de afetos para melhor se compreender, compreender o outro e poder ajudar a pessoa doente a ganhar mais autonomia e lidar com a doença de modo proveitoso para si mesma ${ }^{9}$. Nesse processo, a relação profissional-paciente é muito importante, porque os fluxos de afetos de cada profissional com o usuário e familiares são diferentes, permitindo que as possibilidades de ajudar o sujeito doente sejam maiores, e que esses participem ativamente no tratamento ${ }^{10}$.

Talvez a maior dificuldade encontrada nesse processo de implantação tenha sido o fato de que o comprometimento dos atores é desigual. Tal fato é compreensível em processos de mudança, pois cada sujeito é dotado de valores, crenças e objetivos próprios que, por vezes, sobrepõem os objetivos da coletividade. Dessa forma, com dificuldades e avanços, o curso de Odontologia da UEM segue buscando a formação de profissionais humanizados e habilitados no desenvolvimento e execução da melhor abordagem possível para cada caso, buscando responder às necessidades da população brasileira, tanto na sua prática diária nos consultórios quanto na consolidação do SUS. Esse perfil da formação do aluno vem ao encontro do enfoque defendido na integralidade da atenção à saúde ${ }^{11}$. Além disso, quando os acadêmicos estão mais próximos da população, durante as visitas domiciliares da sua área de abrangência, podem visualizar a realidade das condições de vida das pessoas, com suas diversas limitações (socioeconômicas, geográficas, cognitivas). Isso permite que sejam identificados os problemas e as possibilidades de soluções para se atingir a qualidade de vida ${ }^{12}$. Sem a restrição de olhar apenas a saúde bucal, torna-se mais ampla a análise dos problemas daquele paciente, família ou comunidade, fortalecendo o trabalho focado na intersetorialidade. Claro que essa inserção na comunidade, bem como a atuação clínica, deve ser precedida de um preparo ético e bioético dos acadêmicos, pois, segundo Zoboli ${ }^{13}$, nos encontros de cuidado, estão, no mínimo, duas pessoas (um usuário e um profissional), que são iguais no mérito de respeito; porém distintas quanto às suas biografias e ao momento do processo saúde-doença, requerendo diálogo com base na igual dignidade, para bem cuidar.
O princípio de transversalidade apontado pela $\mathrm{PNH}^{14}$ trata de concepções e práticas que atravessam as diferentes ações e instâncias que aumentam o grau de abertura da comunicação intra e intergrupos e ampliam as grupalidades, o que se reflete em mudanças na prática da saúde. A experiência vivida na CA tem demonstrado o quão valiosa pode ser a pluralidade de grupos envolvidos na comunicação, pois compartilhando do mesmo ambiente e problemas, podem, unidos, transformar a realidade.

\section{CONCLUSÕES}

A clínica ampliada tem dado subsídios para uma nova visão e abordagem para o cuidado integral do usuário da clínica Odontológica da UEM e, assim, melhorar a formação do acadêmico e a atenção prestada à comunidade. Além disso, percebe-se que a construção coletiva agrega muito valor às ações, mas trata-se de um processo lento e que depende da maturidade e comprometimento da equipe de trabalho para que as mudanças de fato ocorram de forma a atender, com êxito, as demandas da sociedade e do Sistema Único de Saúde. Cabe salientar que novas avaliações deverão ocorrer no âmbito da clínica ampliada, com todos os participantes e segmentos envolvidos, em um processo em constante evolução e aprimoramento.

\section{AGRADECIMENTOS}

Aos acadêmicos, docentes e agentes universitários do curso de Odontologia da Universidade Estadual de Maringá que têm se empenhado na implementação e na viabilidade da clínica ampliada, demonstrando a importância e o resultado da construção coletiva.

\section{REFERÊNCIAS}

1. Brasil. Ministério da Saúde. Secretaria-Executiva. Núcleo Técnico da Política Nacional de Humanização. HumanizaSUS: Política Nacional de Humanização: a humanização como eixo norteador das práticas de atenção e gestão em todas as instâncias do SUS / Ministério da Saúde, Secretaria-Executiva, Núcleo Técnico da Política Nacional de Humanização. - Brasília: Ministério da Saúde, 2004.

2. Campos, G. W.S. Saúde Paidéia, Campos, Gastão. São Paulo: Editora Hucitec. 2003.

3. Hayacibara MF, Terada RSS, Takeshita WM (org). Manual da Clínica de Odontologia da Universidade Estadual de Maringá. Maringá: Clichetec, 2010.

4. Neto, JCM. Jornal de poesia. Disponível em: <http: // www.revista.agulha.nom.br/joao02.html> Acesso em: 19 de setembro de 2010, 20: 11 . 
5. Lima RCD. SUS e as teias de diálogos (im)pertinentes para transformar a formação dos trabalhadores de saúde com vistas à humanização das práticas. Interface. 2009; 13(suppl. 1): 789-791.

6. Brasil. Ministério da Saúde. Secretaria de Atenção à Saúde. Núcleo técnico da Política Nacional de Humanização. Clínica Ampliada, Equipe de referência e Projeto Terapêutico Singular / Ministério da Saúde, Secretaria de Atenção à Saúde, Núcleo técnico da Política Nacional de Humanização. - 2. ed. - Brasília: Editora do Ministério da Saúde, 2008.

7. Brasil. Ministério da Saúde. Secretaria de Atenção à Saúde. Política Nacional de Humanização da Atenção e Gestão do SUS. Clínica ampliada e Compartilhada / Ministério da Saúde, Secretaria de Atenção à Saúde, Política Nacional de Humanização da Atenção e Gestão do SUS. - Brasília: Ministério da Saúde, 2009.

8. Campos GWS, Amaral MA. A clínica ampliada e compartilhada, a gestão democrática e redes de atenção como referenciais teórico-operacionais para a reforma do hospital. Ciênc. saúde coletiva. 2007; 12 (4): 849-859.

9. Santos AM, Assis MMA. Da fragmentação à integralidade: construindo e (des)construindo a prática de saúde bucal no Programa de Saúde da Família (PSF) de Alagoinhas, BA. Ciênc. saúde coletiva. 2006; 11 (1): 53-61.

10. Schimith MD, Lima MADS. Acolhimento e vínculo em uma equipe do Programa Saúde da Família. Cadernos de Saúde Pública. 2004; 20 (6): 1487-1494.

11. Ceccim RB, Feuerwerker LCM. Mudanças na graduação das profissões de saúde sob o eixo da integralidade. Cad. Saúde Pública. 2004; 20 (5): 1400-1410.

12. Junqueira LP. Novas formas de gestão na saúde: descentralização e intersetorialidade. Saúde e Sociedade. 1997; 6 (2): 31-46.

13. Zoboli ELCP. Bioética e atenção básica: para uma clínica ampliada, uma Bioética clínica amplificada. O Mundo da Saúde. 2009; 33(2): 195-204.
14. Brasil. Ministério da Saúde. Secretaria de Atenção à Saúde. Núcleo técnico da Política Nacional de Humanização. Grupo de Trabalho de Humanização/ Ministério da Saúde, Secretaria de Atenção à Saúde, Núcleo técnico da Política Nacional de Humanização. - 2. ed. - Brasília: Editora do Ministério da Saúde, 2008.

\section{CONTRIBUIÇÃO DOS AUTORES}

Mitsue fez a concepção ideológica do trabalho e revisão final, Luiz Fernando redigiu o trabalho, Raquel trabalhou na implantação da clínica ampliada e forneceu os dados históricos do Curso, Mirian conduziu o programa de educação tutorial e fez revisão bibliográfica do trabalho, Carina fez avaliação do trabalho na ótica da política nacional de humanização, Hélio coordenou a reestruturação da clínica para implantação da clínica ampliada e revisou dados históricos do departamento, Luciene, Julyana e Arivaldo reestruturaram o setor de triagem transformando-o em setor de acolhimento, além de revisarem o artigo neste aspecto e Cristiane participou na reestruturação da parte operacional da Clínica ampliada e fez a descrição desta para o artigo.

\section{CONFLITO DE INTERESSES}

Declarou não haver.

\section{ENDEREÇO PARA CORRESPONDÊNCIA}

Luiz Fernando Lolli

Universidade Estadual de Maringá - Departamento de Odontologia

Av. Mandacaru, 1550 - Bloco S-08 - Maringá

CEP. 87080 - 000 PR

E-mail: profluizodontologia@uol.com.br 